\title{
System for simultaneous measurements of bilaterally symmetrical acupoints multifrequency impedances
}

This article was published in the following Dove Press journal:

Medical Devices: Evidence and Research

27 November 2013

Number of times this article has been viewed

Mi-Yong Su'

Yong-Hong Tan ${ }^{2}$

Zi-Min Wang ${ }^{3}$

Cai-Jun $\mathrm{Gan}^{3}$

Shou-Hong Chen ${ }^{3}$

'School of Electronic Engineering, Xidian University, Xi'an, Shaanxi, People's Republic of China; ${ }^{2}$ College of Information, Mechanical and Electronic Engineering, Shanghai Normal University, Shanghai, People's Republic of China; ${ }^{3}$ School of Electronic Engineering and Automation, Guilin University of Electronic Technology, Guilin, Guangxi, People's Republic of China
Correspondence: Mi-Yong Su School of Electronic Engineering, Xidian University, No 2, South Taibai Road, Xi'an City 71007I, Shaanxi Province, People's Republic of China Emailsum_yong@126.com

\begin{abstract}
Devices which can obtain comparable bilaterally symmetrical acupoints (BSA) multifrequency impedances (MFI) are often needed in the detection of the energy balance states of acupoints in traditional Chinese medicine. To satisfy these needs, a two-channel impedance measurement system has been introduced which is capable of accurately and simultaneously measuring BSA MFI. The system includes a set of five electrodes, two of which are injected with exciting current signal to synchronously and equally excite BSA; the other three electrodes are used as sensors to simultaneously sense the response signal from both sides. The system also includes a PC-based time-domain signal testing platform with arbitrary current waveform generation and three channels (one exciting current and two response voltages) simultaneously sampling, and a set of MFI simultaneously unbiased computing algorithms based on special odd multisine current signal input. Preliminary validating experiments suggest that the system allows accurate and synchronous measurement of BSA MFI at least in the frequency range of $10 \mathrm{~Hz}$ to $60 \mathrm{kHz}$, and the obtained BSA MFI are well comparable.
\end{abstract}

Keywords: acupuncture, meridians, Qi, balance, two-channel

\section{Introduction}

Traditional Chinese medicine believes that there are 12 primary meridian channels connecting the internal organs, body trunk, and the limbs together. Qi (subtle vital energy) and blood, which is generated in internal organs, is circulated to the whole body by these channels to nourish the body and maintain life. Acupuncture points (acupoints), mostly being located along the 12 channels and being an important component of meridians, "function like miniature electrical relay stations along a vast power line, helping to maintain the flow of energy along each meridian."'1

The morphological structure of each meridian channel is bilaterally geometrically symmetrical to the center of the body (though it may not be exactly symmetrical because of the inexact symmetry of the shape of the body). The locations of acupoints on 12 channels are also bilaterally symmetrical. The two symmetrical acupoints are called bilaterally symmetrical acupoints (BSA), and are always named with the same name. $^{2}$

If the Qi can circulate unblocked to all parts of the body and the level of Qi in BSA is balanced, then the body is healthy. If the Qi in one channel is imbalanced, then the related organs will be prone to illness. ${ }^{3}$

How to reliably measure the balance states of Qi and blood in BSA to acquire the health conditions of internal organs is an important subject in modern study on meridians. 
Electrical impedance is a simple, noninvasive, and effective method to detect the physiological status of biological tissue in modern biomedical engineering. ${ }^{4}$ This method is also a commonly used method to detect the energy balance states of acupoints in traditional Chinese medicine.

Through measurement and comparison of the two resistances of BSA, it is found that the acquired resistance imbalanced values are related to the energy balance states of their corresponding meridian channels and internal organs..$^{5-8}$ A reasonable interpretation of these findings in the viewpoint of modern biomedical engineering is that the imbalanced values result from the physiological differences between the two BSA. ${ }^{4}$ Therefore, these findings can provide scientific evidence for the substantiation of energy balance theory of the meridian, and have the potential to be used to constitute a kind of quantitative and objective index for the diagnosis of internal organ diseases.

To further confirm and apply the relative experimental results, measuring devices which can acquire accurate and comparable BSA multifrequency impedances (MFI) are required. Ryodoraku and electroacupunture According to Voll (EAV) are the two main categories of devices often used..$^{8-11}$ But these devices are inadequate in practical application. From a technical standpoint, the main inadequacies are as follows: ${ }^{8-11}$

1. Since the electrode schemes used for impedance measurements are usually two-electrode techniques in the two devices mentioned above, measurement errors resulting from unstable electrode contact impedances (UECI) are hard to be prevented to invisible level;

2. Less information is obtained, because only direct current resistance or single-frequency impedances are detected;

3. BSA impedances which are asynchronously measured are less comparable because human body pathophysiological states and their corresponding impedances change with time.

In order to overcome the inadequacies above to achieve accurate and comparable BSA MFI, measuring devices capable of simultaneous high-precision measurement of BSA two-channel MFI are needed. However, devices equipped with all these functions are scarce.

The purpose of this paper was, therefore, to study a new measuring system to satisfy these requirements. First, a fiveelectrode technique used for BSA impedances simultaneous measurement was studied. Measuring errors caused by the UECI will be greatly reduced for measurements based on these five-electrode techniques. We also studied a time-based two-channel and MFI simultaneous testing platform, including hardware setup and high-precision MFI computing algorithms. Finally, two validating examples were given.

\section{Electrode schemes for BSA impedances synchronous measurement Definition of BSA impedances}

In Ryodoraku and EAV devices, BSA impedances are defined as the impedances of subcutaneous tissues between the measured acupoints and one palm (right or left). When measuring, the common electrode (reference electrode) is held immobile in one palm, and the measuring electrode is placed on the measured acupoints. ${ }^{5}$ Because the distances between the measured BSA and the common point are not equal, the acquired BSA impedances are not comparable. In this paper, the common point is set on a point on the midline of the body (usually on the acupoint of Dazhui [GV14]) ${ }^{12}$ so the common points are equal in distance from BSA, allowing us to compare BSA impedances with each other.

\section{Five-electrode techniques}

For convenience, BSA equivalent impedances of the upper limbs are noted as $Z_{L A}$ and $Z_{R A}$, respectively, and lower limbs' impedances are noted as $Z_{L L}$ and $Z_{R L}$, respectively. A new BSA impedance synchronous measurement five-electrode technique was presented, which included an exciting electrode $\left(P_{E}\right)$, a ground electrode $\left(P_{G}\right)$, a common electrode $\left(P_{C}\right)$, and two bilateral measuring electrodes $\left(P_{L}\right.$ and $\left.P_{R}\right)$. The electrodes were positioned as follows: $P_{C}$ was placed at the common point; $P_{L}$ and $P_{R}$ were placed at the two BSA to be measured (usually at bilateral Source acupoints); ${ }^{12} P_{E} / P_{G}$ were placed at the acupoints on the meridian line equal to $P_{R} / P_{L}$ at its outer side (usually at Well acupoints). ${ }^{12}$ Alternating constant current is injected between the $P_{E}$ and $P_{G}$ electrodes. Left and right meridian segments are connected in series in the current loop. The current flowing in two meridian segments is synchronous and equal, while two channel voltages are simultaneously measured between $P_{R}$ and $P_{C}$ and between $P_{L}$ and $P_{C}$. Then, according to Ohm's Law, BSA impedances are synchronously detected. The synchronous measuring schematic diagram (Figure 1) demonstrates an example of the measurements of a pair of Source acupoints impedances.

The impedances of any two meridian segments connected in series (such as the meridian lines on forearm and postbrachium) can also be synchronously detected using the 


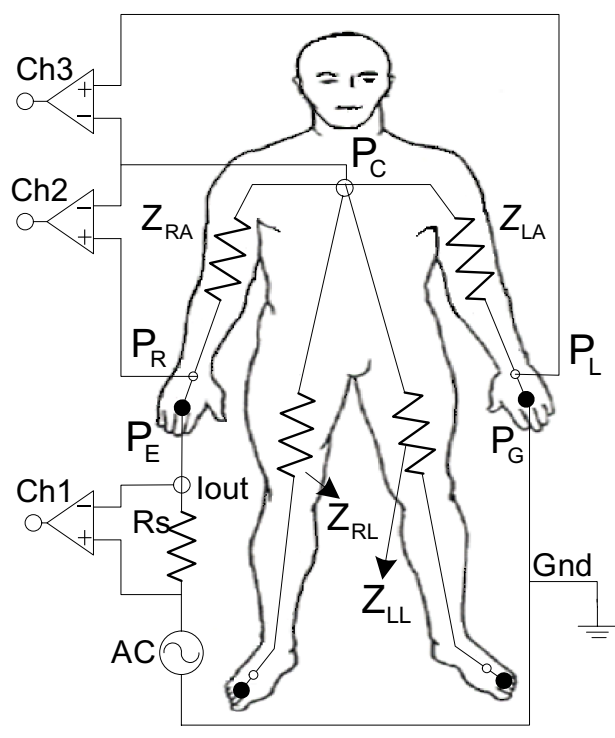

Figure I BSA impedances synchronous measuring schematic diagram. Abbreviations: AC, alternating current source; BSA, bilaterally symmetrical acupoints; $C h$, channel; Gnd, electrical ground; $\mathrm{P}_{C}$, common electrode; $\mathrm{P}_{\mathrm{E}}$, exciting electrode; $P_{G}$, ground electrode; $P_{L}$, left side measuring electrode; $P_{R}$, right side measuring electrode; $R s$, current sampling resistor; $Z_{L A}$, left arm acupoint equivalent impedance (AEI); $Z_{L L}$; left leg $A E I ; Z_{R A}$, right arm $A E I ; Z_{R L}$, right leg $A E I$.

five-electrode schemes mentioned previously. In addition, more bilateral acupoint impedances can be simultaneously measured by adding more bilateral measuring electrodes. Artifacts resulting from the contact impedances between measuring electrodes and tissue are greatly reduced because the exciting electrodes and the measuring ones are not the same. So the five-electrode schemes are extensible and more reliable.

\section{Hardware and software system setup}

Traditionally, there are two kinds of impedance measuring methods: the frequency domain method and the time domain method. ${ }^{13,14}$ MFI are asynchronously measured using the former and synchronously measured using the latter, so the latter usually takes less time than the former. Because BSA electrical properties change over time, the BSA MFI ought to be synchronously and quickly measured in order to improve MFI comparability; the experimental system was designed for this requirement.

The hardware setup, as shown in Figure 2, includes an upper personal computer, a multichannel signal simultaneous sampling card (NI PCI-6115, National Instruments, Austin, TX, USA), arbitrary waveforms generator (Hantek DDS-3005, Hantek, Qingdao, Shandong, People's Republic of China), voltage-controlled current source (basing on the chip of AD844), preamplifier circuits (based on the chip of

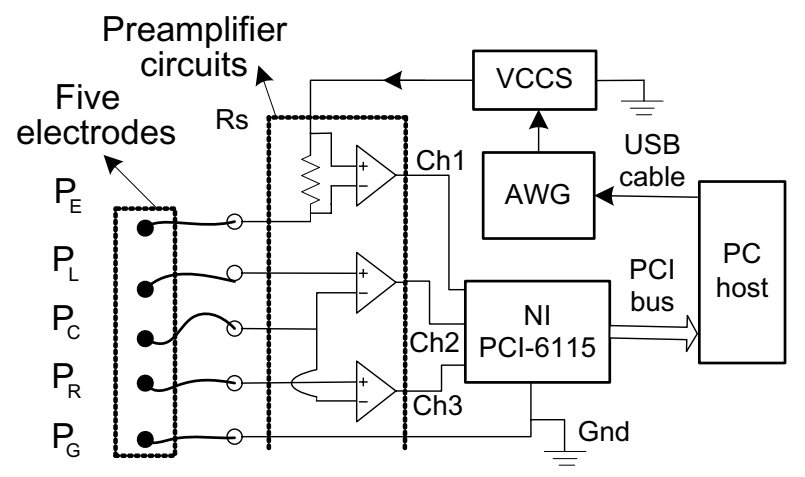

Figure 2 Block diagram of the hardware system.

Abbreviations: AWG, arbitrary waveforms generator; $\mathrm{Ch}$, channel; Gnd, electrical ground; $\mathrm{P}_{\mathrm{C}}$, common electrode; $\mathrm{PC}$, personal computer; $\mathrm{PCl}$, peripheral component interconnect; $P_{E}$, exciting electrode; $P_{G}$, ground electrode; $P_{L}$, left side measuring electrode; $P_{R}$, right side measuring electrode; Rs, current sampling resistor; USB, universal serial bus; VCCS, voltage-controlled current source.

INA116), and five $\mathrm{Ag} / \mathrm{AgCl}$ electrodes. The software platform was developed based on MATLAB 7 DAQ Toolbox (The MathWorks, Natick, MA, USA).

With this system, both BSA response voltages and the exciting current are synchronously sampled. Even these three-channel signals can be continuously sampled if the hard disk's capability is limitless. These functions will improve subsequent high accuracy impedance computation.

\section{BSA MFI simultaneous computing methods}

The exciting signal is selected as a band-limited multisine waveform in which only the amplitudes of special-odd frequency components are nonzero, ${ }^{15}$ as in Equation 1:

$$
i(t)=\sum_{q=0}^{N-1} \sqrt{2}\left[\begin{array}{l}
P_{2 q+1} \sin \left(2 \pi(8 q+1) t / T+\varphi_{2 q+1}\right) \\
+P_{2 q+2} \sin \left(2 \pi(8 q+3) t / T+\varphi_{2 q+2}\right)
\end{array}\right],
$$

where the period of multisine $i(t)$ is $T$ and $i(t)$ contain $2 N$ sinusoids; $P_{k}$ and $\varphi_{k}$ are root mean square and phases of the $k$ th sinusoid, respectively; $k=1, \ldots, 2 N, P_{k}$ are set to be equally positive numbers; and $\varphi_{k}$ is the result of minimization of the peak factor of $i(t) .{ }^{16}$ Compared with the waveforms of square, rand, and PRBS, the signal of Equation 1 has the advantages of no spectra leak (integer period sampling), less interharmonic distortion, easy acquisition of high signal-tonoise ratio, and so on.

In reality, the sampled exciting signal and response signal are unavoidably subjected to random noise and the effect of gain mismatches among different channels (GMADC). Highly accurate MFI are hard to be calcu- 
lated directly with Ohm's law. To realize a highly accurate calculation of MFI, these two kinds of noises must be eliminated. According to the advantages of the exciting signal, multicycle averaging denoise algorithm and channel-gain-error-compensated method were adopted to achieve a highly accurate calculation of MFI, as seen in Equation 2 (in frequency domain):

$\hat{\mathrm{Z}}(j \omega)=\frac{\frac{1}{M} \sum_{m=1}^{M} \tilde{\mathrm{V}}_{m}(j \omega)}{\frac{1}{M} \sum_{m=1}^{M} \tilde{\mathrm{I}}_{m}(j \omega)}=\frac{\overline{\mathrm{V}}(j \omega)}{\overline{\mathrm{I}}(j \omega)} \cdot \mathrm{H}_{\mathrm{T}}(j \omega)$

where $\tilde{\mathrm{V}}_{m}(j \omega)$ and $\tilde{\mathrm{I}}_{m}(j \omega)$ are the spectra of $m$ th periods of observed exciting current and output voltage, respectively; $\mathrm{H}_{\mathrm{T}}(j \omega)$ is the ratio of the gains of voltage channel to the gains of current channel; and $\overline{\mathrm{I}}(j \omega)$ and $\overline{\mathrm{V}}(j \omega)$ are averages of $M$ periods of current and voltage contaminated by random noise (no GMADC effects), respectively.

Methods to eliminate two kinds of errors in Equation 2 are as follows:

1. For the random noise: The larger the $M$ is, the lower level the random noise is reduced to, and the longer the testing time. In reality, $M$ will be selected to satisfy both the prescribed testing time and acceptable noise level.

2. For the GMADC effects: To determine $\mathrm{H}_{\mathrm{T}}(j \omega)$, a known standard resistor $R_{0}$ is used as device under test. The result is $\hat{Z}_{\mathrm{C}}(j \omega)=R_{0} \cdot \mathrm{H}_{\mathrm{T}}(j \omega)$. Then $\mathrm{H}_{\mathrm{T}}(j \omega)=\hat{\mathrm{Z}}_{\mathrm{C}}(j \omega) / R_{0}$. Finally, the $\mathrm{H}_{\mathrm{T}}(j \omega)$ is used to calibrate the left side part of Equation 2; the result is

$$
\bar{Z}(j \omega)=\hat{Z}(j \omega) / \mathrm{H}_{\mathrm{T}}(j \omega) \approx \mathrm{Z}(j \omega)
$$

where $Z(j \omega)$ is the real impedance value. Thus, an unbiased measurement of MFI is obtained. Note that the right and left channel MFI are calibrated using its own $\mathrm{H}_{\mathrm{T}}(j \omega)$, respectively.

\section{Validating experiment \\ Resistor-capacitor circuit}

Right and left channels of the proposed system were used to measure the MFI of a resistor-capacitor (RC) circuit consisting of a resistor $\mathrm{R}_{\mathrm{e}}(1200 \pm 12 \Omega)$ in parallel with a series combination of a resistor $R_{i}(8.2 \pm 0.082 \Omega)$ and a capacitor $\mathrm{C}_{\mathrm{m}}(0.1 \pm 0.005 \mu \mathrm{F})$. The wiring diagram is shown in Figure 3. A multisine current signal, as presented in Equation 1, with equal nonzero amplitudes on the

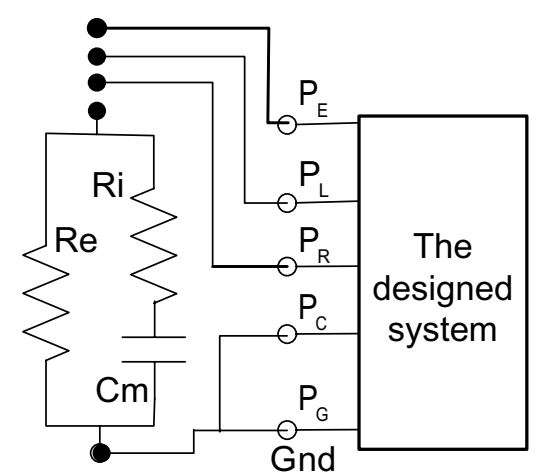

Figure 3 Wiring diagram of impedance measurement of a RC combinatorial circuit. Abbreviations: BSA, bilaterally symmetrical acupoints; $\mathrm{Cm}$, capacitor; Gnd, electrical ground; MFI, multifrequency impedances; $P_{C}$, common electrode; $P_{E}$, exciting electrode; $P_{G}$, ground electrode; $P_{L}$, left side measuring electrode; $P_{R}$, right side measuring electrode; RC, resistor-capacitor; Re, resistor I; Ri, resistor 2.
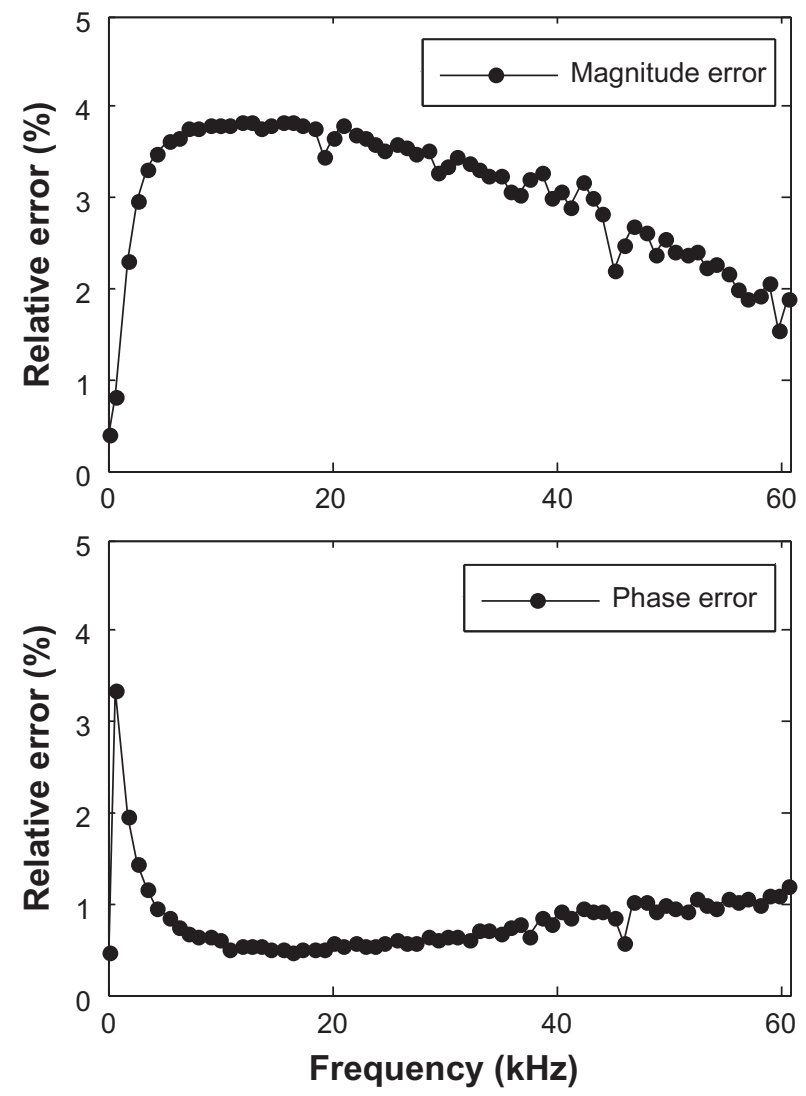

Figure 4 Magnitude and phase relative error between RC circuit normal impedances and impedances measured by the left channel.

Abbreviation: RC, resistor-capacitor.

frequency points of $[1,3,9,11, \ldots, 6056,6059] * 10 \mathrm{~Hz}$ and with time domain amplitude range of $-25 \sim+25 \mu \mathrm{A}$, was used as stimuli. Sampling frequency is $2 \mathrm{MHz}$. The values of $\mathrm{H}_{\mathrm{T}}(j \omega)$ were measured using the same stimuli before impedance measurement of RC circuit. Fifteen periods of stimuli and corresponding stable response signals (sampling time, 1.5 seconds) were sampled and 

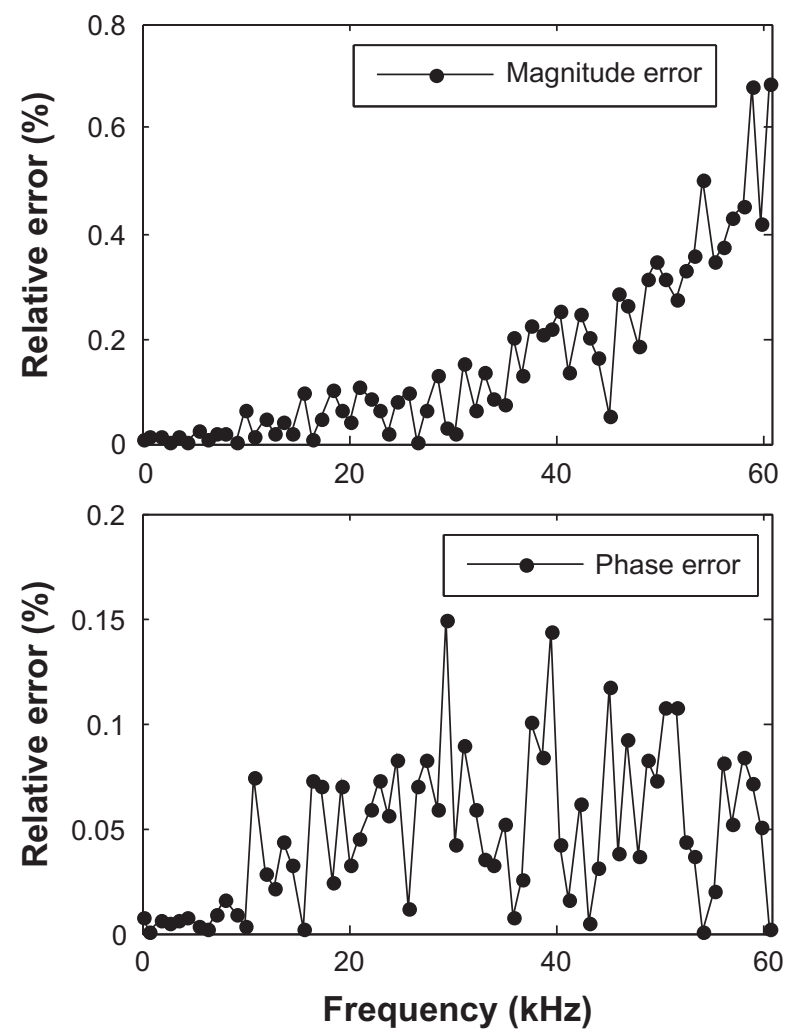

Figure 5 Magnitude and phase relative error between two impedances measured by the left and right channels.

were used to calculate the MFI as shown in Equations 2 and 3. The results are presented in Figures 4 and 5, where the diagram of testing errors is shown to illustrate system measuring accuracy.

The maximum relative errors of magnitude and phase in Figure 4 were $3.9 \%$ and $3.4 \%$, respectively, which were less than the accuracy of the capacitor (5\%). In Figure 5, the maximum relative errors of magnitude and phase were $0.7 \%$ and $0.15 \%$, respectively, which are lower than the accuracy of the resistor $(1 \%)$.

The results in Figure 4 illustrate that the left channel possesses a fairly high measuring accuracy. The results in Figure 5 indicate that the two impedances measured by left and right channels were almost the same, and implied that the magnitude-frequency characteristics of the two testing channels were calibrated to be almost the same. The experimental data also show that relative error curves between normal impedances and impedances measured by the right channel were almost the same as the one in Figure 4, so the right channel relative error curves were not repeated there. The correspondence between the magnitude-frequency characteristics of the two measuring channels, and their fairly high measuring accuracy, indicates that the two channels can achieve comparable results.

\section{Bilateral acupuncture points of Yangxi (LI 5)}

One of the functions of acupoint of Yangxi is that it can be used to treat Rhinitis. Two females, one healthy 32-year-old and one 36-year-old patient with rhinitis, were invited as participants.

The five electrodes were positioned as follows: $P_{E}$ and $P_{G}$ on the right and left side acupoints of Shangyang (LI1), respectively; ${ }^{12} P_{C}$ on Dazhui; and $P_{L}$ and $P_{R}$ on the right and left side acupoints of Yangxi, respectively (see Figure 1). The stimuli, sampling frequency, and sampling duration were as listed in Section 5.1. Before measurement, relative skin sites were cleaned with alcohol, followed by $0.9 \%$ $\mathrm{NaCl}$ solution. The results are shown in Figure 6 (healthy patient) and Figure 7 (rhinitis patient). The maximum relative errors of magnitude and phase in Figure 6 were 1.1\% and $17.1 \%$, respectively. The maximum relative errors of magnitude and phase in Figure 7 were 5.5\% and 18.5\%, respectively. These results show that imbalanced values between impedances of BSA of Yangxi in the rhinitis patient are greater than those in the healthy patient. It further indicates that the proposed measuring system is very beneficial to accurately detect imbalanced values between impedances
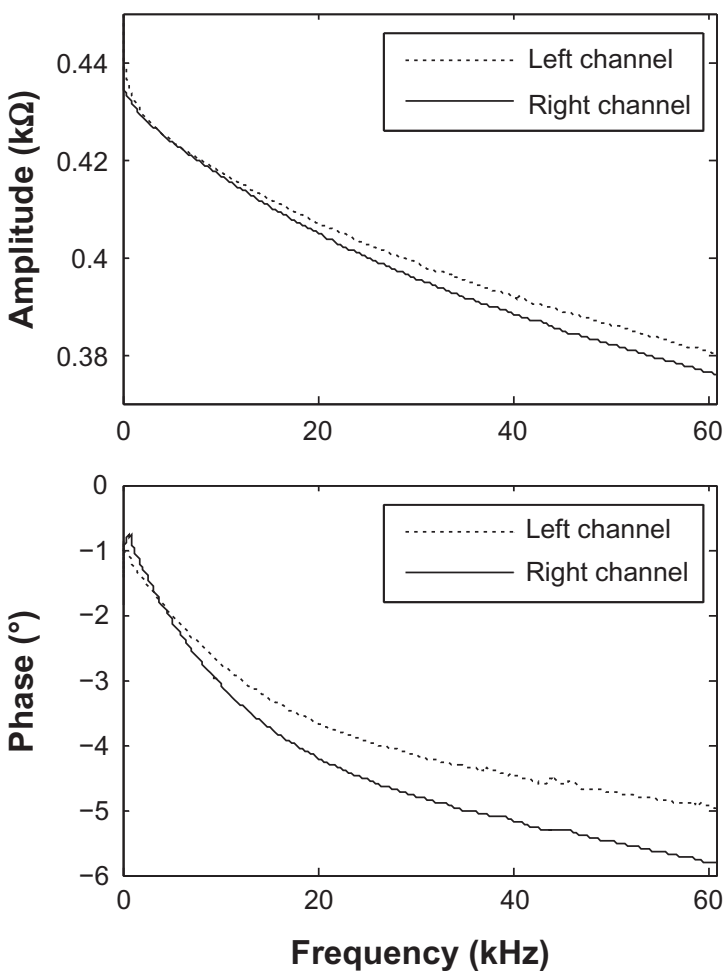

Figure $6 \mathrm{MFI}$ of BSA of Yangxi of a 32-year-old healthy woman measured by the proposed system.

Abbreviations: BSA, bilaterally symmetrical acupoints; MFI, multifrequency impedances. 

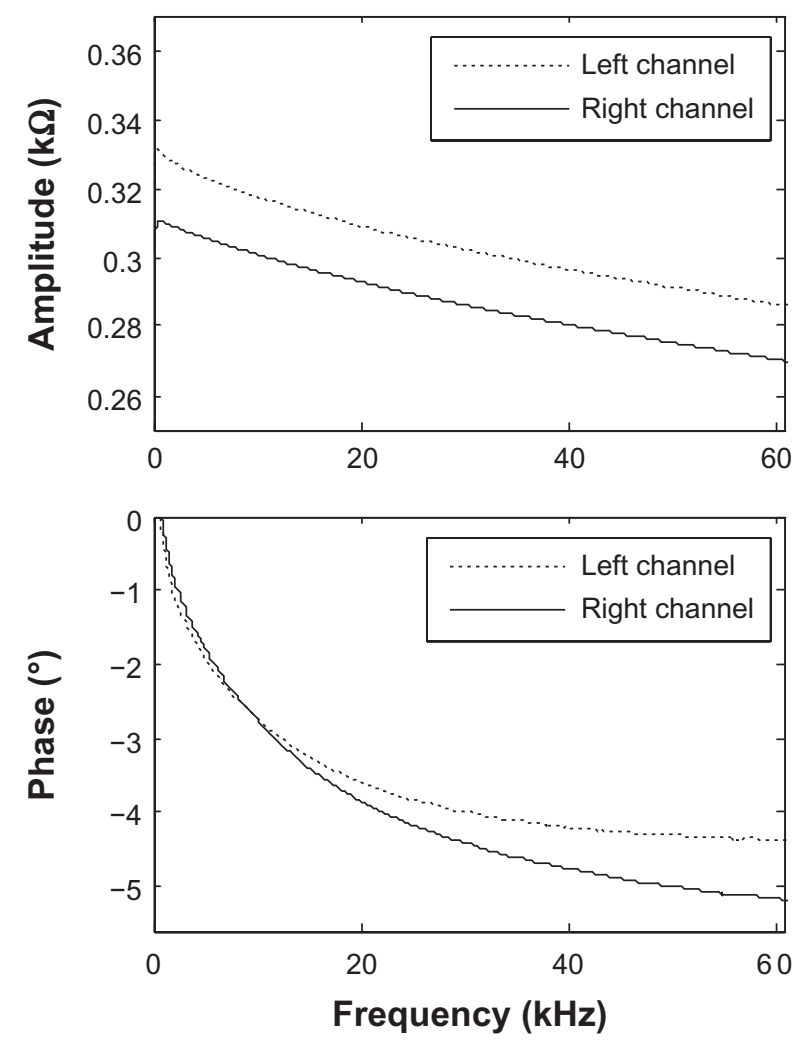

Figure $7 \mathrm{MFI}$ of BSA of Yangxi of a 36-year-old female patient with rhinitis. Abbreviations: BSA, bilaterally symmetrical acupoints; MFI, multifrequency impedances.

of BSA, and that these values can stand for some kind of physiological status.

\section{Discussion}

Because not all current flows along the measured meridian channels, the acquired results are a kind of impedance of a variety of tissues, including measured meridian channels, and reflect a kind of macroscopic electrical property between measured points and the common point. How to limit the exciting current to flow only through the meridian line is still an open problem. But compared with two-electrode, ${ }^{8}$ fourelectrode ${ }^{17,18}$ or multielectrode methods, ${ }^{19,20}$ the presented five-electrode methods have the advantages of being able to realize the excitation of BSA synchronously and equally, and reliably sense their response signals with less effect from UECI.

This system provides unbiased measurement, according to the result of theoretical derivation presented in Section 4. But actual measurements are biased, and the bias is prone to become larger as the measuring frequency increases, as seen in Figure 5. It is affected mainly by the factors of stray capacitance in circuit system, nonlinear distortion coming from oversized stimuli, unstable direct current power supply system, and so on. All these factors should be fully considered to satisfy the requirements of measurement precision in limited measuring time. In addition, postdigital signal processing is another effective way to acquire more precise measurements.

Validating experiments indicated that the system can measure impedances from $10 \mathrm{~Hz}$ to $60 \mathrm{kHz}$, which cover the impedance frequency range many studies need. , $^{2,-11,17-20}$ According to the hardware configurations in Section 3, our system has the potential to measure impedances up to $1 \mathrm{MHz}$ through further optimizing signal amplification circuit board layouts and other modifications. For the need to measure impedances above $1 \mathrm{MHz},{ }^{21,22}$ the proposed system still falls short. Compared to the devices used in other studies, ${ }^{2,4-11,17-22}$ our system has the advantage of being able to measure BSA MFI exactly and simultaneously.

More experiments and theoretical analysis are needed to address how to study an imbalanced indicator, which can provide more information on the nature of differences and how the imbalanced indicators are related to internal organs physiological states.

\section{Conclusion}

The new designed BSA impedance measuring system includes: 1) a new five-electrode scheme used for BSA impedances synchronous measurements and less UECI error measurements; 2) a time-based MFI synchronous testing platform which is capable of arbitrary current waveform generation and a three-channel signal (an input current and two response voltages) simultaneous sampling; and 3) a set of MFI simultaneous unbiased computing algorithms based on special odd multisine current signal input. Preliminary validating experiments suggest that the designed system can synchronously and accurately measure BSA MFI at least in frequency range of $10 \mathrm{~Hz}$ to $60 \mathrm{kHz}$, and compensate for relative technical defects in existing devices. The BSA impedances measured by the designed system are thus more comparable, and the information extracted from them is more abundant and reliable. This system is a reliable impedance testing tool for the study of the relation between energy balance states of BSA and the physiological status of internal organs.

\section{Acknowledgment}

This work is partly supported by the National Science Foundation of China (NSFC Grant no 60971004).

\section{Disclosure}

The authors report no conflicts of interest in this work. 


\section{References}

1. Tova Navarra. The Encyclopedia of Complementary and Alternative Medicine. New York: Facts on File; 2004.

2. Lu WA, Tsuei JJ, Chen KG. Preferential direction and symmetry of electric conduction of human meridians: Bilaterally symmetrical acupoints provide better conductance for a better "connection". IEEE Eng Med Biol Mag. 1999;18(1):76-78.

3. Zhou YP, Wang KM, Hu L, et al. Meridian-viscera correlationship. In: Ying X, Ding GH, Wu GC, editors. Current Research in Acupuncture. New York: Springer; 2013:559-599.

4. Halter RJ, Hartov A, Heaney JA, Paulsen KD, Schned AR. Electrical impedance spectroscopy of the human prostate. IEEE Trans Biomed Eng. 2007;54(7):1321-1327.

5. Ahn AC, Schnyer R, Conboy L, Laufer MR, Wayne PM. Electrodermal measures of Jing-Well points and their clinical relevance in endometriosis-related chronic pelvic pain. J Altern Complement Med. 2009; 15(12):1293-1305.

6. Chamberlin S, Colbert AP, Larsen A. Skin conductance at 24 Source (Yuan) acupoints in 8637 patients: influence of age, gender and time of day. J Acupunct Meridian Stud. 2011;4(1):14-23.

7. Huang SM, Chien LY, Chang CC, Chen PH, Tai CJ. Abnormal gastroscopy findings were related to lower meridian energy. Evid Based Complement Alternat Med. 2011;2011:878391.

8. Tsuei JJ. The science of acupuncture-theory and practice. IEEE Eng Med Biol Mag. 1996;15(3):52-57.

9. Prokhorov EF, Prokhorova TE, González-Hernández J, et al. In vivo dc and ac measurements at acupuncture points in healthy and unhealthy people. Complement Ther Med. 2006;14(1):31-38.

10. Ahn AC, Martinsen OG. Electrical characterization of acupuncture points: technical issues and challenges. J Altern Complement Med. 2007;13(8):817-824.

11. Weng CS, Tsai YS, Yang CY. Using electrical conductance as the evaluation parameter of pain in patients with low back pain treated by acupuncture-like TENS. Biomed Eng Appl Basis Commun. 2004; 16(4):205-212.
12. WHO Regional Office for the Western Pacific. WHO Standard Acupuncture Point Locations in the Western Pacific Region. Manila: World Health Organization; 2008.

13. Pliquett U. Time-domain based impedance measurement: strengths and drawbacks. J Phys Conf Ser. 2013;434(1):012092.

14. Gerald W, Klaus RN, Erich S. Fast impedance spectroscopy: general aspects and performance study for single ion channel measurements. Rev Sci Instrum. 2000;71(6):2309-2320.

15. Vanhoenacker K, Schoukens J, Guillaume P, Vanlanduit S. The use of multisine excitations to characterise damage in structures. Mech Syst Signal Pr. 2004;18(1):43-57.

16. Guillaume P, Schoukens J, Pintelon R, Kollar I. Crest-factor minimization using nonlinear chebyshev approximation methods. IEEE Trans Instrum Meas. 1991;40(6):982-989.

17. Zhang WB, Jeong DM, Lee YH, Lee MS. Measurement of subcutaneous impedance by four-electrode method at acupoints located with single-power alternative current. Am J Chin Med. 2004;32(5): 779-788.

18. Ahn AC, Wu J, Badger GJ, Hammerschlag R, Langevin HM. Electrical impedance along connective tissue planes associated with acupuncture meridians. BMC Complement Altern Med. 2005;5:10.

19. Thong T, Colbert AP, Larsen AP. An 8-channel skin impedance measurement system for acupuncture research.Conf Proc IEEE Eng Med Biol Soc. 2009;2009:861-864.

20. Fukumoto T, Ohba S, Futami R. A basic study about multi channel measurement of skin impedance vector loci on the acupuncture points. In: 23rd Annual EMBS International Conference. October 25-28, 2001. Istanbul, Turkey. Abstract 3392-3395.

21. Teplan M, Kukučka M, Ondrejkovičová A. Impedance analysis of acupuncture points and pathways. J Phys Conf Ser. 2011;329: 012034.

22. Egot-Lemaire SJ, Ziskin MC. Dielectric properties of human skin at an acupuncture point in the $50-75 \mathrm{GHz}$ frequency range: a pilot study. Bioelectromagnetics. 2011;32(5):360-366.
Medical Devices: Evidence and Research

\section{Publish your work in this journal}

Medical Devices: Evidence and Research is an international, peerreviewed, open access journal that focuses on the evidence, technology, research, and expert opinion supporting the use and application of medical devices in the diagnosis, treatment and management of clinical conditions and physiological processes. The identification of novel

\section{Dovepress}

devices and optimal use of existing devices which will lead to improved clinical outcomes and more effective patient management and safety is a key feature. The manuscript management system is completely online and includes a quick and fair peer-review system. Visit http://www. dovepress.com/testimonials.php to read real quotes from authors. 